\title{
8 Zusammenfassung
}

Es war das Ziel dieser Abhandlung, die Entwicklung der Vereinigten Staaten zwischen 1865 und 1900 als Abfolge von Prozessen darzustellen, die weder mit zwanghafter Notwendigkeit noch vollkommen zufällig zur hegemonialen Stellung geführt haben, die die USA nach 1898 eingenommen haben. Diese Entwicklung hätte auch anders verlaufen können. Es gibt in der Geschichte keine Linearität und keine Notwendigkeit. Geschichte ist ein Prozess mit vielen Faktoren, die alle miteinander verbunden sind und sich alle gegenseitig beeinflussen. Da Menschen Mangelwesen sind, die für ihre Existenz und ihr Überleben bestimmte materielle Voraussetzungen benötigen, gibt es zwar eine starke Tendenz, die Erreichung dieser Voraussetzungen als Explanans menschlichen Handelns zu sehen, doch sind die materiellen Voraussetzungen vielfach vermittelt, werden von ideologischen, religiösen, politischen, kulturellen Faktoren geglättet und aufgeworfen, abgeschwächt oder verstärkt. Materielle Voraussetzungen sind zudem historisch wandelbar und sind über sozioökologische Rückkopplungsmodelle gesellschaftlich beeinflussbar. Der Weg der Vereinigten Staaten von Amerika vom Dissens des Bürgerkriegs hin zum aggressiven Nationalpatriotismus des ausgehenden 19. Jahrhunderts war geprägt durch Schwellen, sogenannte Lock-ins, die der Theorie der Pfadabhängigkeit entsprechend, eine Lösung einer bestimmten, begrenzten Problemlage herbeiführten, die nicht unbedingt die logisch beste oder wünschenswerte Lösung darstellte, die aber zum Ausgangspunkt weiterer Problematiken wurde, die nach einer Phase der Latenz und Unentschiedenheit sich wieder verdichteten und einer Lösung zugeführt wurden. Im Laufe des hier präsentierten historischen Narrativs habe ich neun solcher Kristallisationspunkte benannt. Der erste lag in Präsident Andrew Johnsons „Programm der Versöhnung“, das von einer möglichst raschen Reintegration der alten Eliten des Südens in die politischen Strukturen der wiedervereinten Union ausging. Johnson ging es um die nationale Einheit. Die Belange von rund vier Millionen Sklavinnen und Sklaven, die keinen Besitz und kein Einkommen hatten, waren ihm dabei gleichgültig. Damit wurde die Bühne bereitet für eine Kooperation der alten und neuen Eliten des Südens und des Nordostens. Versuche der „radikalen“ Republikaner, diese Grundsatzentscheidung rückgängig zu machen, scheiterten am entschiedenen Widerstand der alten Landbesitzerelite im Süden, die in der Lage war, Teile der weißen Bevölkerung im Süden für ihre Interessen zu mobilisieren und so das Programm der radikalen Republikaner zu verhindern.

Der „Kampf im Süden“ kulminierte schließlich im resignierten Abzug der USArmee aus dem Süden (Lock-in 2), wodurch die Südstaatendemokraten und die mit ihnen verbundenen suprematistischen Terrortruppen wie der KKK und andere 
Verbände freie Hand bekamen und die Republikaner und die Freedpeople Schritt für Schritt zurückdrängen konnten, bis der Süden „solide“ geworden war, im Sinne der Alleinherrschaft einer politischen Partei.

Es bedurfte zur Beendigung der Rekonstruktionspolitik des Kongresses aber mehr als des Einsatzes der Demokraten zur Herbeiführung eines ApartheidSystems im Süden. Die Republikanische Partei war im doppelten Sinne die Partei Lincolns. Sie hatte eine Anti-Sklavereitradition, aber sie hatte auch deutliche Bezüge zu einer wirtschaftsfreundlichen Haltung, die die Tradition der in den Republikanern aufgegangenen Whigs fortführte. Um die Reconstruction zum Scheitern zu bringen, war es auch nötig, die Republikaner zu einer Partei der Unternehmer umzuformen, ein Prozess, der dadurch erleichtert wurde, dass ein Großteil der Bevölkerung des Nordostens nach 1873 andere Probleme hatte als die Emanzipation der ehemaligen Sklavinnen und Sklaven. Klassenkämpfe, wirtschaftliche Not, hervorgerufen durch die rasche Industrialisierung des Nordens, Massenimmigration und der Verlust der Kontrolle über den Arbeitsprozess prägten die Diskurse der Jahre nach der Wirtschaftskrise von 1873. Die Abwendung der Republikaner und eines großen Teils der Wählerschaft von den Problemen der Reconstruction sorgte für die Zweiteilung der Nation in einen agrarischen Süden und einen industriellen Nordosten. Das Ende der Reconstruction (Lock-in 3) stellt jene Bifurkation dar, die es den Republikanern erlaubte, sich auf das „Kerngeschäft“ der Industrialisierung zu beschränken und den Süden den national unerheblichen Demokraten zu überlassen.

Der Eisenbahnbau von Investoren des Ostens, die Landgeschenke der Bundes- und Staatenregierungen erhielten, um den Bau von Eisenbahnen zu finanzieren, sowie der Landhunger von prospektiven Farmerfamilien führten zu einer aggressiven Landnahme in jenen Teilen des US-Territoriums, in dem noch große Gruppen von Native Americans lebten. Der Siedlerimperialismus, der in der Vertreibung der Native Americans nach 1865 resultierte (Lock-in 4), stellte eine notwendige Voraussetzung für diese Landnahme dar. Die indigene Bevölkerung musste nicht nur die ökonomische und rechtliche Kontrolle über ihr Land verlieren, sie musste auch reloziert werden, wobei die Regierung, Armee und Eisenbahngesellschaften nicht davor zurückschreckten, Genozide zu begehen, indem sie die Lebensgrundlage der indianischen Gruppen zerstörten (Massentötungen der Büffel) und immer wieder Massaker an indianischen Gruppen verübten. Ohne Vertreibung der Indianer und Enteignung ihres Landes wäre der Eisenbahnbau, wie er betrieben wurde, nicht möglich gewesen.

Die Eisenbahn (Lock-in 6) prägte als Schlüsselindustrie und Motor des Industriekapitalismus die Entwicklung des amerikanischen Wirtschaftswesens in der Phase nach dem Bürgerkrieg, da andere Schlüsselindustrien (Eisen, Stahl), die Entwicklung des Agrarwesens (exportorientierter Rohstoffanbau im Süden, 
Mittleren und Fernen Westen) und die demographischen Verschiebungen (Zuzug von Immigranten und Immigrantinnen) vom Ausbau des Eisenbahnwesens direkt abhingen. Gleichzeitig generierte die Eisenbahnindustrie die Exzesse der Spekulation, die 1873 und 1893 zu zwei schweren und langwierigen Rezessionen führten, die arbeitende Menschen an den Rand des Ruins brachten.

Die dem Kapitalismus inhärente Tendenz zu Konzentration konnte zwar auch in England, Frankreich und Deutschland etwa zeitgleich beobachtet werden, doch zeichnete sich die amerikanische Entwicklung dadurch aus, dass die Rechte von Individuen, wie sie während der Reconstruction definiert worden waren, auf die wirtschaftlichen Körperschaften übertragen wurden, weshalb eine wirkungsvolle Interessenvertretung der arbeitenden Menschen ausgehebelt werden konnte. Dieser Lock-in-Effekt (Lock-in 6) führte zu einem Wettbewerbsvorteil der Unternehmer, da Gewerkschaften durchweg als „Verschwörung“ oder Streiks als Eingriff in die Vertragshoheit der Unternehmen definiert werden konnten.

Verstärkt wurde diese Form der rechtlichen und ökonomischen Diskriminierung durch das System des Rassismus, das Weiß gegen Schwarz, Eingewanderte gegen Alteingesessene und Englisch sprechende Menschen gegen die polyglotte Masse des „Rests“ stellte. Dieser Lock-in (Lock-in 7) baute auf dem System der rassistischen Suprematie des Südens auf, perpetuierte und exportierte es unter den Bedingungen der Industrialisierung in den Norden und konnte hervorragend instrumentalisiert werden, um Arbeitskämpfe auszubremsen oder aufzuweichen. Aus rassistischen Gründen diskriminierte Arbeiter und Arbeiterinnen konnten als Streikbrecher eingesetzt werden. Die an Rassenzuordnungen ausgerichtete Politik eines großen Teils der Facharbeitergewerkschaften stützte dabei dieses System nachhaltig. In den Diskussionen um den Eintritt der USA in ein koloniales System des Empire diente dieser internalisierte Rassismus als Rechtfertigung der imperialen Ansprüche der USA gegenüber people of color.

Dieser Rassismus machte auch vor den Organisationen der Arbeiterbewegung nicht halt. Er manifestierte sich hier in vierfacher Hinsicht, als alltägliche Praktiken des Ausschlusses, als Diskurse der Minderwertigkeit und der Unangepasstheit, innerhalb von Institutionen, die diese Diskurse in Entscheidungen umsetzen (Nichtaufnahme von Hilfsarbeitern, Minderheiten und Frauen), und in Gesetzen wie dem Chinese Exclusion Act, die auf allen diesen Faktoren aufbauten und sie in einem Feed-Back-Loop verstärken: Die Arbeiter- und Gewerkschaftsbewegung wurden so auf eine expansionistische und chauvinistische Generallinie vorbereitet, die in den Jahren nach 1893 und im Zusammenhang mit der Diskussion um den Erwerb amerikanischer Kolonien an Akzeptanz gewinnen sollte.

Alle Lock-ins zusammengenommen in ihrer Sequentialität bedingen die letzte Koagulation der amerikanischen Geschichte des 19. Jahrhunderts, den Spanisch- 
Amerikanischen Krieg von 1898. Er ist Endpunkt einer Entwicklung von Verfestigungen in der Innenpolitik, zugleich Ausgangspunkt der Ausbildung einer globalen Rolle der USA im 20. Jahrhundert. Der Spanisch-Amerikanische Krieg stellt das Scharnier dar, das die innere Entwicklung der USA und die Bedeutung der Vereinigten Staaten als ökonomische, militärische und kulturelle Vormacht des 20. Jahrhunderts miteinander verbindet. Ohne diesen Krieg hätten die USA wahrscheinlich weder Puerto Rico noch die Philippinen erworben. Ohne diese Territorien hätte die Rolle der USA im Fernen Osten im Hinblick auf China und Japan anders ausgesehen. Ohne das Steckenpferd der kontrafaktischen Geschichte hier zu Schanden zu reiten, kann man behaupten, dass der splendid little war den Aufstieg der USA abschloss und dem europäischen Kolonialismus old style die Todesglocke läutete. Der Kolonialkrieg auf den Philippinen zeigt schließlich, dass die Stereotype des indian-hating und des anti-schwarzen Rassismus auch unter den Bedingungen eines militärischen Engagements außerhalb der USA wirksam und bedeutungsvoll blieben. 